\title{
MALESTAR EN LA(S) INFANCIA(S)
}

Alicia Muniz Martoy

Doctora en Psicología de la USAL Profesora titular de la Facultad de Psicología de la UDELAR Miembro Habilitante de AUDEPP

Profesora titular del IUPA Correo electrónico: munizalicia55@gmail.com.uy ORCID: 0000-0002-3508-0907 


\section{Resumen}

El artículo se propone problematizar la situación actual de las infancias en nuestro país, en relación a la patologización de las conductas. Advierte acerca de las prácticas y discursos en torno a los modos de abordar las dificultades que se presentan tanto a nivel del aprendizaje como a nivel conductual. Hace hincapié en la comprensión compleja del sufrimiento infantil, enmarcado en los modos de vinculación intrafamiliar. Ofrece conclusiones extraídas de una investigación académica y propone críticamente alternativas para su abordaje clínico.

Palabras clave: infancia, vínculo intrafamiliar, patologización.

\section{Discontent in Childhood(s)}

\section{Abstract}

This article aims at problematizing the current situation of childhoods in our country, in relation to the pathologization of behaviors. It warns about the practices and discourses around the ways of approaching the difficulties that arise both at the learning level and at the behavioral level, emphasizing the complex understanding of childhood suffering, framed in the modes of intra-family bonding. It offers conclusions drawn from an academic investigation and critically proposes alternatives in their clinical approach.

Keywords: chilhood, intra-family bond, pathologization. 
Dani é uma criança que não sabe andar de bicicleta. Todas as crianças do seu bairro já andam de bicicleta; os da sua escola já andam de bicicleta; os da sua idade já andam de bicicleta. Foi chamado um psicólogo para que estude seu caso. Fez uma investigação, realizou alguns testes (coordenação motora, força, equilíbrio e muitos outros); falou com seus pais, com seus professores, com seus vizinhos e com seus colegas de classe e chegou a uma conclusão: esta criança tem um problema, tem dificuldades para andar de bicicleta. Dani é 'disbiciclético'. Agora podemos ficar tranqüilos, pois já temos um diagnóstico. Agora temos a explicação: o garoto não anda de bicicleta porque é disbiciclético e é disbiciclético porque não anda de bicicleta.

Emílio Ruiz Rodriguez (2009, párr. 1)

El fenómeno no es nuevo. Novedosa ha de ser la mirada sobre la(s) infancia(s) (sostenemos el plural dado que no puede considerarse que haya una infancia homogénea), perspectivas que los clínicos debemos afinar en torno a las manifestaciones del sufrimiento en niños, niñas y adolescentes que llegan hasta el consultorio.

¿Será que acaso las teorías resultan obsoletas, será que los que llegan traen cuadros nuevos o que aparecen nuevas patologías con manifestaciones en la infancia antes nunca vistas? Si así fuera, el derrumbe de los estratos teóricos podría dejar a los más avezados terapeutas en un estado de vulnerabilidad sin parangón... Si así fuera, los niños y niñas se nos presentarían tan extraños que correríamos el riesgo de declararnos incapaces de comprender el fenómeno que se presenta.

En este artículo proponemos mirar allende el niño y la niña que nos convocan; mirémonos como adultos, como hacedores de discursos que 
impregnan miradas y prácticas cotidianas en cualquiera de nuestros roles: padres, madres, maestros, educadores, médicos, psicólogos, terapeutas, psicomotricistas, fonoaudiólogos y tantos otros que trabajan en pro de un mejor desarrollo de aquellos que muestran el fracaso ante la inadaptación al sistema.

Malestar(es) en la cultura... ¿De qué cultura hablamos cuando nos habita el malestar? Echemos un vistazo sobre nuestras condiciones de producción (de)subjetivante.

\section{PANDEMIANDO}

En tiempos de incertidumbre, pandemia y otras novedosas modalidades de construirnos en ciudadanía, nos topamos con una situación que de golpe ha puesto en suspenso nuestras vidas o, al menos, las formas en que nuestras vidas venían siendo vividas hasta el momento. Niños, niñas y adolescentes han quedado sin la presencia de amigos, de abuelos, de maestros, de terapeutas, de apoyos..., en definitiva, de aquello que necesitaba de los cuerpos para ser efectivo. Inventamos entonces un nuevo uso de dispositivos que allí estaban, como esperando su gran oportunidad, para mostrar su capacidad (limitada) de acercamiento. Al menos es algo.

Mientras tanto, la vida está volviendo a una pretendida normalidad si como tal se entiende el dar cabida a las actividades que, en un año recién estrenado, habían quedado detenidas. Volvieron las clases, pero en casa, a través de dispositivos que hasta ahora servían sobre todo para jugar y que caían bajo la reprimenda de adultos por su uso y abuso. Ahora se volvieron imprescindibles. Claro, no para todos es posible el acceso por igual. No todos tienen la misma oportunidad. Algunos igualmente quedarían por el camino, algunos quedarían en stand by, esperando el rescate de lo presencial. No obstante, aún estamos esperando 
los encuentros, aquello que pone en juego los cuerpos en un espacio compartido. La alegría del entre-nos, la alegría de darle un susto al miedo al contacto.

La reducción de la vida al espacio privado con gran relevancia de lo íntimo, mientras lo público está acotado, el acuñar nuevos lenguajes que por momentos generaban dudas sobre su eficacia (cuarentenar, hisopar, confinar), el uso de tecnologías nuevas en la vestimenta (barbijo, tapabocas, guantes de látex), son formas que exigen gran esfuerzo psíquico. Mantener la distancia física, pero no la social, es el gran desafío.

Ambientes privados fértiles para el recrudecimiento de las locuras internas, pero también para el aprendizaje, en la mutualidad, de otros modos de convivencia. Mantener la calma, la cordura, los principios y los cuidados está siendo la mayor de todas las exigencias; para no decaer, para no sucumbir, sobre todo porque nadie tiene idea de cuánto tiempo va a ser necesario sostener esto. Vivimos, ensayamos, cambiamos, volvimos a probar mientras seguimos viviendo.

Los psicoterapeutas no quedamos por fuera de tales desafíos, sacudidos de nuestros lugares cómodos en la intimidad del consultorio: improvisamos, inventamos, probamos, desechamos formas de intervención que nos impidieran mantener intacto nuestro oficio. $\mathrm{O}$ al menos eso nos creemos. Los pacientes..., mejor de lo que pensamos.

Los niños y las niñas se acomodaron más fácilmente de lo que tuvimos que acomodar nuestro cuerpo inmóvil frente a las pantallas. Nos metimos en sus casas, conocimos a las mascotas, los dormitorios, sus juguetes, la cocina familiar, tratando de sostener al máximo el espacio incontaminado de la sesión. Aprendimos mucho, escuchamos y vimos mucho más, ellos nos orientaron por dónde seguir. «¿Tenés por ahí una tijera y un papel?», «¿Podrás hacer un dibujo?», preguntaron..., como si pedir algo más, bajo estas circunstancias del encuentro, fuera una irreverencia. 
Mientras tanto, nuestros espacios privados también fueron expuestos. Intercambiamos privacidades, se nos metieron en casa los pacientes - grandes y chicos - y les exigieron a los nuestros compartir el hogar con ellos.

\section{PENSAR LA(S) INFANCIA(S) EN SU (MAL) ESTADO}

El desvío precedente se debe a que no podríamos escribir sin una contextualización, sin dejar en claro por dónde pasan las afectaciones en el aquí y ahora del tiempo que transitamos.

Aún no sabemos si lo que se expone hoy tiene vigencia, habrá que esperar. Mientras tanto, ensayamos algunos pensamientos que nos orienten en torno a los modos de (mal) estar en nuestra cultura, a la vez que nos pensamos como clínicos de la(s) infancia(s) y adolescencia(s).

Ante la dispersión de las condiciones de producción actuales, recortar un fragmento de una realidad implica, siguiendo el pensamiento de Lewkowicz (2004), generar una situación capaz de ser pensada aun en las condiciones más adversas. Esto no significa desconocer el origen desde el cual surgen, sino que la visión crítica sobre las condiciones de producción para esa situación son las que aportarán elementos para ser pensable.

En oportunidades anteriores (Muniz Martoy, 2011, 2013), expusimos acerca de los fenómenos clínicos infantiles a la luz de las circunstancias actuales de relacionamiento entre la medicina y la vida cotidiana. La psicología clínica se ha enfrentado en el último tiempo al desafío de comprender los fenómenos complejos del psiquismo infantil, fenómenos que escapan a las descripciones clásicas y a las teorizaciones hegemónicas. Lo clínico es lo singular, justamente por eso no puede ser reductible de manera fácil.

Se afirmaba entonces que la(s) infancia(s) se presentan de forma diversa. Los niños y las niñas (Bleichmar, s. f.) aparecen con un sinnúmero 
de características y, si no se abordan desde una perspectiva compleja, se corre el riesgo de patologizar rápidamente conductas que responden a los modos actuales de convivencia, de cotidianeidades no estables; en definitiva, de condiciones de subjetivación novedosas que se encarnan en la cultura misma.

Sostenemos que la concepción de los clínicos acerca del armado de la producción subjetiva provee de elementos para establecer hipótesis acerca del porqué del enfermar (hacer síntomas) y acerca de las estrategias adecuadas para intervenir sobre ese sufrimiento a fin de producir alivio y bienestar, a fin de potencializar las capacidades sociales, cognitivas $\mathrm{y}$ afectivas del sujeto.

No resulta indiferente partir de uno $u$ otro marco referencial para entender los fenómenos en cuestión. Un paradigma positivista podría reducir elementos altamente complejos, como el psiquismo infantil, a su expresión neurobiológica, mientras que el paradigma complejo permitiría trabajar sobre hipótesis de diferentes niveles, que son suplementarias y no excluyentes, de manera simultánea. Faraone et al. (2009) señala, también, que tales clasificaciones derivadas de discursos donde predomina la lógica del signo no son ingenuas, que remiten a posicionamientos ideológicos, políticos y económicos. Por su parte, Vasen (2009) expresa, en tal sentido, que se puso énfasis en utilizar el traje bipolar para el cuerpo infantil. A ello sumamos nuestra percepción del tironeo y de los remiendos aplicados, que comienzan a surgir cuando esto no da cuenta estrictamente de las singularidades en la infancia.

\section{VIOLENCIA, SI LAS HAY EN TALES PRÁCTICAS}

No deberíamos mantenernos indiferentes ante estos fenómenos y sus efectos, dado que las categorías de salud y enfermedad son producto 
de un tiempo y valores sociales. Pero, además, son altamente sensibles a políticas de salud estatales, intereses económicos y posibilidades de existencia (de marcas, de ofertas, de servicios, de discursos). Todos estos ingredientes arman la cultura a través de los íconos, discursos y prácticas que se derivan, en la que nos movemos los terapeutas y los pacientes, usuarios, consultantes. El cambio en la nominación de estas prácticas no siempre implica un cambio profundo en la mirada sobre el sufrimiento. A veces es solo un nombre para que no cambie la lógica con la que se piensan.

Integrar la dimensión histórica se vuelve imprescindible, ya que los modos de vincularidad (intrafamiliar y comunitaria) presentan directa correlación en la producción de síntomas y en los modos disfuncionales de cotidianeidad familiar y en presencia de eventos traumáticos. Untoiglich (2011) afirma que los niños y niñas estudiados por déficit atencional tienen dificultad para poner en palabras sus sentimientos, conflictos, tensiones, y buscan su expresión a través de la descarga motora. Esto es también lo que sucede a nivel familiar: se han encontrado familias que guardan celosamente secretos innombrables, situaciones traumáticas silenciadas. Parece obvio que la conducta del niño o niña se relacione directamente con estas consignas (no siempre explícitas) a nivel familiar, pero no es suficiente aún para explicar la presencia del déficit atencional. La clínica encuentra un cuerpo infantil sujetado a exigencias institucionales (de la familia, de la escuela, de la salud) que no siempre acompañan las necesidades de los niños y niñas actuales.

Cuando desconocemos la importancia de los vínculos intrafamiliares y la incidencia que tienen a la hora de producir salud o enfermedad, se comete un error grave, en tanto se está dejando al niño en solitario, a cargo del llamado déficit o trastorno como única causa y único efecto. El niño o la niña señalado, sancionado, excluido en su singularidad bajo las formas del disciplinamiento medicamentoso, las penitencias, la 
desafiliación escolar o la derivación a escuelas especiales, queda instalado en un borde de riesgo, por más tratamientos que el Estado le provea. El apoyo social tiene efecto directo sobre la salud y la vivencia de bienestar; tal es así que, cuando el apoyo se expresa en función del rasgo «niño enfermo que necesita ayuda», se le quita el reconocimiento como sujeto deseante, como identidad singular. En la clínica, estos pacientes llegan a presentarse como «Soy un bipolar, soy un ADD», discurso que sostiene la captura de la identidad integral del sujeto a partir de un rasgo que, en este caso, es patologizante, lo que deja una marca a modo de estigma que lo acompañará largo tiempo, al menos durante su trayectoria curricular.

Volnovich (2017), en su maravilloso prólogo del texto Infancias, de Stavchansky y Untoiglich, dice:

nuestros niños son nuestra Historia. Cada generación se apropia de la Historia al advenir a ella, y encarna los mitos de las que la preceden. Nuestros niños como Historia nuestra son testigo-testimonios de un proyecto genocida, de una empresa de exterminio y en cada síntoma, en el más banal de los síntomas del menos neurótico de nuestros niños, hablan del espanto y la tragedia que amenaza a repetirse a cada paso [...] estamos marcados por los mismos horrores. (pp. 13-14)

Los niños cuentan la Historia de nuestro tiempo, de su devenir, a la vez que son futuros posibles. Entonces: ¿por qué condenarlos bajo una mirada reduccionista, simplista, inhóspita del sufrimiento que encarnan? Al decir «nuestro tiempo», nos referimos a lo que construimos en conjunto, al orden de lo social, lo común-comunitario, imposible de silenciar y dejar por fuera de nuestras hipótesis. 
Continúa Volnovich (2017):

Así, hoy en día, el psicoanálisis cumple con el delicado trabajo [...] de hacerle frente al dolor y al sufrimiento que la adaptación al sistema no sólo no ha logrado atenuar, sino que aporta como plus, como malestar en la cultura. Hoy en día el espacio de la clínica debería estar al servicio de la imaginación, de la denuncia de la naturalización del consumo; al servicio de reforzar la esperanza de poder transitar este mundo con valor crítico y poder transformador. (p. 16)

Son tiempos de hegemonías discursivas, de mandatos de bienestar y de eficiencias, que los adultos reproducimos por temor al fracaso, temor a no poder prepararlos lo suficiente para lo que viene: para las exigencias. Tiempos de neurociencias al servicio del neuroliberalismo, que nos transforma en consumidores de drogas para una mayor eficacia en la máquina productiva: para seguir y para parar, para estar y para evadirse, para despertarse y para dormirse en tiempo y forma.

$\mathrm{La}(\mathrm{s})$ infancia(s) no están exentas de estos discursos y prácticas, de la mano de sentencias tan repetidas como «para que baje las revoluciones», «para que pueda aprender», "para que aguante el doble horario».

Las cifras de niños y niñas medicamentalizados y con polifarmacia en nuestro país son alarmantes, al igual que en el mundo desarrollado (Giachetto, 2019).

Pero ello no lo es todo, porque las derivas de estas lógicas llevan a modalidades de clasificación, según la capacidad para estar en la escuela o para aprender. ${ }^{1}$ Niños y niñas que no pueden responder a los

1 En el momento de escribir el presente artículo, se está presentando en Uruguay un proyecto de ley para la creación de un registro de niños y niñas en edad escolar con diagnóstico de TDAH, diagnosticado por neuropediatra. Fundamenta ello la necesidad de una enseñanza especial basada en métodos conductistas, que obliga a maestros a una capacitación específica y a la adecuación edilicia para su atención (Schusman Kraft, 2020). 
requerimientos del sistema educativo actual deben pagar peaje para estar: tomar las gotitas, ir poco rato, tener doble escolaridad (común y especial).

La investigación Impacto a nivel de los vínculos intrafamiliares del diagnóstico y el tratamiento por dificultades en la atención y por hiperactividad en niños escolares (Muniz Martoy, 2017) ha tenido resultados que demuestran que el sufrimiento se instala en varios niveles. La distancia entre lo esperado y lo obtenido mediante la medicación instala una ambivalencia respecto al tratamiento farmacológico, que se expresa como un no deseo de medicar al niño o niña, a la vez que una pasiva aceptación por hacerlo, en el entendido de que es favorable sobre todo frente a las presiones escolares. Ello produce conductas en las que diferentes actuaciones (actings) revelan la oposición a medicarlo, se generan olvidos o bien suspensiones con diversos motivos, no todos compartibles.

Los datos obtenidos en la investigación referida indican que en la vida familiar esto se vivencia como una imposición de la institución escolar, que exige estar pendientes de la medicación, una erogación económica y un enfrentarse a la mirada del docente y de otros del entorno, que genera angustia (Muniz Martoy, 2017). Esta situación configura una vivencia de malestar, vivida como «una preocupación más» al decir de los padres, que determina ciertos ajustes en la convivencia familiar.

Los padres tienden a proyectar fuera de la familia las causas de lo que les pasa a sus hijos, con escaso insight en cuanto a preguntarse qué tiene que ver con la familia esto que le sucede al niño. Perciben un escaso o nulo contacto con aspectos intrafamiliares en torno a condiciones de crianza o de relacionamiento. Sostienen la fantasía de medicar a sus hijos e hijas solo en los primeros años de la escuela, sin asociar otros cambios necesarios para que esto se vea favorecido. Manifiestan no estar de acuerdo con mantener la medicación a futuro, consistente con la ambivalencia y desconfianza respecto a la figura del médico tratante. 
Ante los cambios percibidos a partir de la medicación, los padres estudiados hablan del impacto que provoca un hijo o hija cambiado, que es vivido como ajeno y no puede ser ligado a la representación del niño o niña que se tenía hasta el momento. Asimismo, este niño cambiado a partir de la medicación, en sentido negativo, provoca angustia en tanto los padres no desean tener que enfrentarse a ello por contemplar las exigencias de la escuela (Muniz Martoy, 2017).

La noticia del hijo o hija portador de un trastorno, tanto como la percepción de un hijo o hija cambiado por los efectos del tratamiento, no es aceptada por la familia. Esto promueve variadas conductas de ambivalencia y contradicción respecto a la adherencia al tratamiento farmacológico. Ello indica el rechazo a incorporar la idea de un hijo o hija diferente a lo representado, a la vez que se percibe con frustración y dolor el efecto que tiene la conducta del niño o niña a nivel extrafamiliar.

En dicha investigación, afirmábamos que

la falta de seguimiento del tratamiento, así como la suspensión y reintegro de la medicación a criterio de los padres constituyen elementos de riesgo para el niño. Esto tampoco permite conocer si el tratamiento es efectivo o no. Para la familia se vuelve intolerable el efecto de indiferencia y desconexión que la medicación provocó en algunos niños, remitiendo a la desinvestidura del entorno que tampoco les permite aprender. (Muniz Martoy, 2017, p. 223)

El tratamiento únicamente farmacológico produce cambios que son vividos por los padres como insuficientes. Pero además lo es para los psicólogos, en tanto percibimos que se coloca en el niño o niña la causa del malestar familiar, con lo cual se evita que el grupo pueda contactar con las circunstancias de inestabilidad, violencia, pérdidas y un largo etcétera por las que transitan las familias de nuestras latitudes. 
El niño, la niña, son el centro de un discurso que proviene de la institución escolar que señala el fracaso o la imposibilidad y los coagula en ese lugar en soledad, sin problematizar las condiciones que así lo producen.

Si el malestar en la posición hijo puede asociarse al malestar del resto de los integrantes de la familia por fuera de la lógica causa-efecto, el camino se abre hacia numerosas posibilidades de pensarse como protagonistas de un sufrimiento que los involucra. El alivio, la solución, también proviene del contexto y no solamente de los cambios en el niño o niña a través de la medicación. Esto sería pedirle mucho...

Por su parte, los niños y niñas que fueron estudiados junto a sus padres muestran, a través de las técnicas proyectivas —Dibujo de la Familia Kinética Actual y el Test de Pata Negra, utilizados en la investigación (Muniz Martoy, 2017)—, una imagen de sí frágil, con fallas en los mecanismos adaptativos que protejan al yo de las exigencias pulsionales del ello. Las figuras realizadas indican la precariedad en la constitución de la imagen de sí mismo destacada en la representación de lo corporal, con alteraciones que van de lo grave a la ausencia misma. El cuerpo como centro de tensión y de alivio a la vez canaliza la descarga de sus tensiones internas por el polo motor.

Janin et al. (2004) refieren que, cuando el objeto falla, se produce una retirada de la investidura, que va al vacío en una búsqueda caótica donde no se diferencian estímulos, lo que provoca desorganización de la acción. La tan mencionada hiperactividad implica una falla en el proceso de inhibición de la impulsividad por fragilidad en el yo y se asocia a dificultades en la simbolización. Por eso, las dificultades académicas quedan asociadas a la hiperactividad con la desatención: no es la inquietud ni la distracción la que produce bajo rendimiento, sino que son fallas de un nivel más estructurante del psiquismo del infans. 
En los niños y niñas estudiados, la representación de lo parental mantiene características de fragilidad también, lo que da cuenta así de las vivencias de poco sostén de sus ansiedades, fantasías y angustias.

Como efecto de los discursos, tan arraigados, que promueven la eficiencia y el exitismo, estos niños y niñas estudiados muestran claramente el elemento estigmatizante con el que se identifican, lo que evidencia que la percepción de sí mismos coincide con lo que el entorno les devuelve. El lugar que el niño percibe que tiene en la familia y en la escuela está en relación con las vivencias de sí respecto a su conducta, caracterizada en general por la desvalorización, la estigmatización y la justificación del castigo por sentimientos de culpa. Esto no es un tema menor, pues las actuaciones (incluso contra sí mismos) responden a estas fantasías.

Para finalizar, transcribimos aquí las principales conclusiones del estudio mencionado:

Se propone pensar entonces en el impacto [del diagnóstico y del tratamiento farmacológico] como un acontecimiento que se instala en la familia y que no puede ser fácilmente semantizado porque es algo vivido como ajeno, impuesto, que no tiene que ver con la autopercepción que como familia tienen de sí mismos. El entorno les devuelve un diagnóstico a manera de imagen que dice acerca de las falencias en uno de sus miembros, el más débil. Se requiere de un trabajo complejo para representar a este nuevo niño que dista del hijo deseado. Esto los enfrenta a una situación inesperada relacionada con el temor a la exclusión de los ámbitos naturales: la escuela y el grupo social inmediato.

En función de lo expresado en esta tesis, se torna imprescindible instrumentar dispositivos terapéuticos que incluyan la dimensión vincular cuando de niños con estas dificultades (y eventualmente otras) se trata, ya que una única alternativa terapéutica no favorece la circulación de sentido a la 
interna de la familia. Sin un abordaje de la perspectiva familiar de las afectaciones del niño, este se queda en soledad a cargo de lo enfermo, lo loco, lo intolerable, y tiene que enfrentar las presiones de las instituciones solo. La familia como productora de subjetividad y protagonista en los procesos del padecer y del curar, tiene un rol trascendental en cuanto a descentrar la mirada del niño patologizado. Los actores de la salud no deberían hacerse eco de discursos simplificantes en tanto el objetivo del trabajo está puesto en la complejidad de la vida misma. (Muniz Martoy, 2017, p. 226)

Post scriptum: Dedico estos pensamientos a Felipe, el más nuevo de la familia, quien llegó a este mundo en medio de estas escrituras, en medio de la pandemia, jen medio de un mundo que vale la pena disfrutar aún!

\section{$\S$}

\section{REFERENCIAS BIBLIOGRÁFICAS}

Bleichmar, S. (2001, 4 de octubre). La infancia y la adolescencia ya no son las mismas. Qué se conserva hoy de la infancia que conocimos [conferencia]. Consejo de los Derechos de Niñas, Niños y Adolescentes, Centro Cultural San Martín. www.elpsicoanalitico.com.ar/num3/ autores-bleichmar-infancia-adolescencia.php

Faraone, S., Barcala, A., Bianchi, E. y Torriccelli, F. (2009). La industria farmacéutica en los procesos de medicalización/medicamentalización en la infancia. Revista Electrónica Margen, 54. www.margen. org/suscri/margen54/faraone.pdf 
Giachetto, G.(2019, 24 demayo).Despatologización delasdiferenciasenniños, niñas y adolescentes [mesa redonda]. Ciclo de Difusión del Programa de Problemáticas Clínicas de la Infancia y Adolescencia del Instituto de Psicología Clínica, Facultad de Psicología, Universidad de la República. www.youtube.com/watch?v=KoamyYmbSLU\&t=2339s Janin, B., Frizzera, O. T., Heuser, C., Rojas, M. C., Tallis, J. y Untoiglich, G. (2004). Niños desatentos e hiperactivos. ADD/ADHD. Reflexiones críticas acerca del Trastorno por Déficit de Atención con o sin Hiperactividad. Noveduc.

Lewkowicz, I. (2004). Pensar sin Estado. Paidós.

Muniz Martoy, A (2011). Vínculos y déficit atencional. Dimensiones de análisis para su comprensión [trabajo final del seminario de Psicología Social, doctorado en Psicología]. Universidad del Salvador, Buenos Aires.

Muniz Martoy, A. (2013). Abordajes clínicos de las problemáticas actuales en la infancia. Psicología, Conocimiento y Sociedad, 3(2), 135154. http://revista.psico.edu.uy/index.php/revpsicologia/article/ view/165

Muniz Martoy, A. (2017). Impacto a nivel de los vínculos intrafamiliares del diagnóstico psicopatológico y del tratamiento por dificultades en la atención y por hiperactividad en niños escolares [tesis doctoral]. Universidad del Salvador, Buenos Aires. https://racimo.usal.edu.ar/5569/

Ruiz Rodriguez, E. (2009, 1 de enero). Disbicicléticos. Inclusive: inclusão e cidadania. www.inclusive.org.br/arquivos/2853

Schusman Kraft, J. (2020, 14 de julio). Trastorno déficit atencional e hiperactividad (TDAH). Registro niños edad escolar. Creación [proyecto de ley]. Cámara de Representantes. https://parlamento.gub.uy/ documentosyleyes/ficha-asunto/147141 
Untoiglich, G. (2011). Versiones actuales del sufrimiento infantil. Noveduc. VASEN, J. (2009). El mito del niño bipolar. Noveduc.

Volnovich, J. C. (2017). Prólogo. En L. Stavchansky y G. Untoiglich, Infancias: entre espectros y trastornos (pp. 13-16). Paradiso. 
